\title{
Prevalence and impact of musculoskeletal pain among staff working in a large integrated sexual health service in UK
}

\author{
S Malik', S Sundaram ${ }^{1}$, S Samraj ${ }^{1}$, J Turner ${ }^{1}$, Prof. Karen Walker-Bone ${ }^{2}$ \\ ${ }^{1}$ Department of Genitourinary Medicine, Royal South Hants, Southampton, UK \\ 2University of Southampton, Southampton, UK
}

\section{Introduction:}

Musculoskeletal pain is common and contributes to sickness absence among people of working age. Although there is published data on this in other specialities, little is known about the incidence of musculoskeletal pain in staff working in sexual health. ${ }^{1}$

\section{Methods:}

We conducted a staff survey through a paper and online questionnaire for staff working across different sexual health units in Solent NHS Trust. Data were collected on any musculoskeletal pain or symptoms affecting various joints including spine, severity of symptoms and their impact on personal life, work and leisure. We also collected data on repetitive tasks undertaken during sexual health work including procedures.

\begin{abstract}
Results:
Our response rate was $49 \%$ (39 completed questionnaires returned out of 80). One staff member who reported pre-existing musculoskeletal problems was excluded. $92 \%$ respondents were female reflecting the workforce demographic in this speciality. $61 \%$ of doctors and $85 \%$ of nurses reported musculoskeletal pain.
\end{abstract}

In those who reported having musculoskeletal symptoms, most commonly it was single site pain $(46 \%)$ and within this group lower back $(54 \%)$ was the most common site. Including those who reported symptoms from multiple sites most common sites of involvement were lower back (57\%), neck pain (35\%) and shoulder pain (35\%).

Low back (LB) pain was more common in nurses (76\%) than doctors $(27 \%)$. However, involvement of single or multiple sites and overall impact were comparable for both groups. Those with or without pain were not significantly different in terms of age, median time working in sexual health or types of routine procedures.

The impact of this pain seems to be quite disproportionate with moderate to fairly severe impact reported for work/daily routine (34\%), social activities/hobbies (29\%), sleep (29\%), fatigue/low energy (26\%) and emotional well being (23\%). Symptoms leading to seeking professional health advice, using medication to achieve control, missing work and interference with normal and recreational activities the most common sites implicated were low back ache, neck and hand pain in that order. Overall impact was reported moderate to fairly severe in $37 \%$ staff. Most commonly reported intensity of pain was moderate- $29 \%$ during day and $46 \%$ during night.

Majority of the respondents had a very good understanding of their condition and its management. The incidence of pain reported by staff undertaking $>10$ implants/week, $>10$ coil insertions per week or $>10$ speculum examinations/week or $>10$ Cryotherapy sessions/week was $100 \%, 83 \%, 77 \%$ and $66 \%$ respectively.

\section{Most common sites of pain where multiple sites were involved}

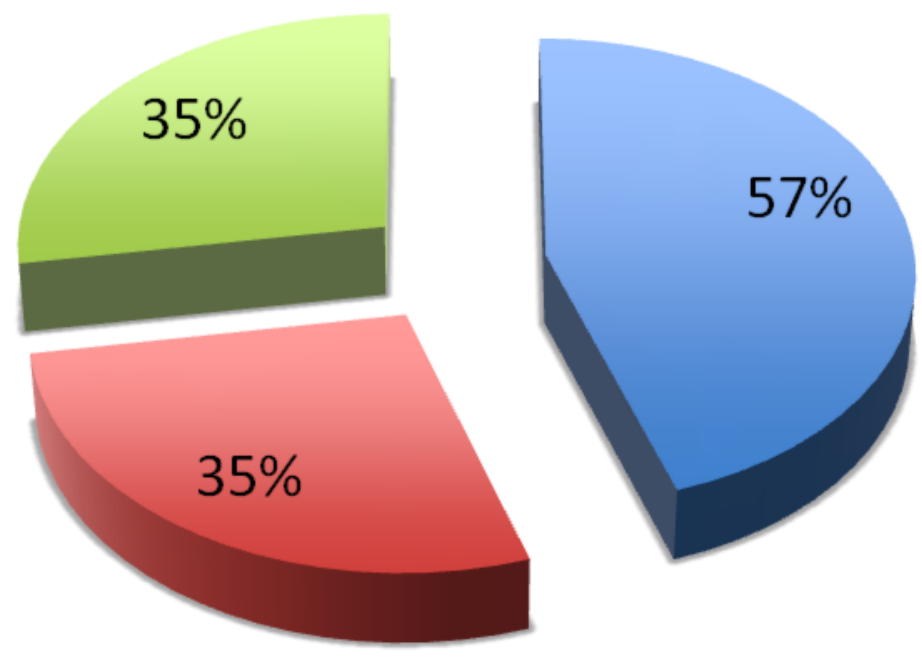

- Lower back

Neck pain

Shoulder pain

\section{Impact of pain}

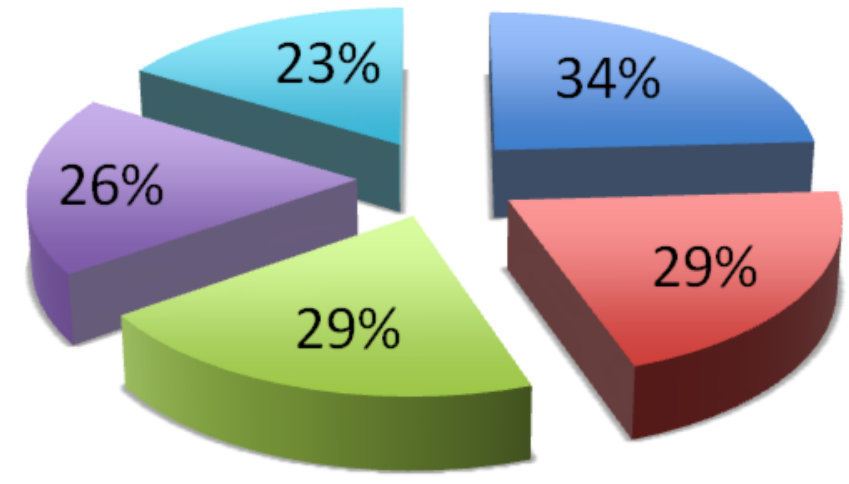

Work/daily routine

Social

activites/hobbies

sleep

Fatigue/low energy levels

Emotional well being

\section{Discussion:}

This study benchmarks the incidence, sites, impact and severity of musculoskeletal problems encountered amongst sexual health staff. These symptoms seem to have a major impact on both professional and personal lives of our staff. Although a higher incidence was noted amongst nurses compared to doctors, no clear reasons could be identified.

\section{Reference:}

1. K Walker-Bone, C Copper. Hard work never hurt anyone: or did it? A review of occupational associations with soft tissue musculoskeletal disorders of the neck and upper limb. Ann Rheum Diss 2009 Jul;68(7):1170. 